\title{
Promoção de Resiliência por meio da Tecnologia a partir das Instituições Científicas, Tecnológicas e de Inovação no Brasil
}

\author{
Promoting Resilience through Technology from Scientific, Technological and Innovation \\ Institutions in Brazil
}

\author{
Silvio Bitencourt da Silva ${ }^{1}$ \\ Daniel Pedro Puffal ${ }^{2}$ \\ Taís Müller Flores ${ }^{3}$
}

\begin{abstract}
Resumo
Este trabalho teórico-empírico busca examinar como a tecnologia promove resiliência, descrita como a meta-capacidade de lidar com situações adversas por meio de estratégias adaptativas, proativas e reativas, a partir de Instituições Científicas, Tecnológicas e de Inovação (ICT's) no Brasil. Esta investigação é pautada em uma abordagem qualitativa e concentra-se em um único estudo de caso com múltiplas unidades de análise, além de ter como base a Aliança Estratégica para a Promoção da Inovação Tecnológica, integrada pela Associação Brasileira das Instituições de Pesquisa Tecnológica e Inovação (Abipti), pela Associação Nacional de Pesquisa, Desenvolvimento e Engenharia das Empresas Inovadoras (Anpei) e pela Associação Nacional de Entidades Promotoras de Empreendimentos Inovadores (Anprotec). Ou seja, a pesquisa se faz a partir das principais entidades que discutem e representam as ICT's no Brasil, apoiada por uma análise jurídica das diferentes normas e documentos de referência que regulamentam as atividades de ciência, tecnologia e inovação no ordenamento jurídico brasileiro. As evidências obtidas demonstram que as ICT's podem promover a resiliência em condições que permitam a sua operação no modelo de hélice quadrupla/quíntupla onde o governo, as empresas, a academia, a sociedade e o ambiente possam se conectar a partir das ICT's em relações colaborativas, semelhantes à noção de inovação aberta que envolve o uso de fluxos de entrada e saída de conhecimento para acelerar a inovação, para o desenvolvimento de soluções, além do escopo que quaisquer organizações ou pessoas que fariam individualmente. Entretanto, os processos colaborativos são difíceis de desencadear e mantendo-se sem políticas que assegurem um ambiente cultural e institucional de apoio às ICT's. Dessa forma, há, de fato, a emergência de um tipo de ICT 4.0 que promova resiliência por meio da tecnologia para enfrentar os novos desafios da chamada quarta revolução industrial que produzem uma era de disrupções, expressa por dinâmicas que se refletem em novas tecnologias, modelo de negócios, produtos e estratégias em empresas e/ou startups que visam sacudir uma indústria e alterar seus padrões competitivos.
\end{abstract}

Palavras-Chave: Colaboração. Disrupção. Instituição Científica. Tecnológica e de Inovação. Tecnologia. Resiliência.

\begin{abstract}
This theoretical-empirical work seeks to examine how technology promotes resilience, described as the meta-capacity to deal with adverse situations through adaptive, proactive, and reactive strategies, based on Scientific, Technological, and Innovation Institutions (SCls) in Brazil. This investigation is based on a qualitative approach. It also focuses on a single case study with multiple units of analysis, in addition to being based on the Strategic Alliance for the Promotion of Technological Innovation, integrated by the Brazilian Association of Technological Research and Innovation Institutions (Abipti), by the National Association for Research, Development, and Engineering of Innovative Companies (Anpei) and by the National Association of Entities Promoting Innovative Enterprises (Anprotec). In other words, the research is based on the main entities that discuss and represent SCls in
\end{abstract} Graduação em Administração e do Mestrado Profissional em Gestão e Negócios na UNISINOS. 
Brazil, supported by a legal analysis of the different standards and reference documents that regulate science, technology, and innovation activities in the Brazilian legal system. The evidence obtained demonstrates that SCls can promote resilience under conditions that allow their operation in the quadruple/quintuple helix model where government, business, academy, society, and the environment can connect from the SCIs in collaborative relationships, similar to the notion of open innovation that involves the use of input and output flows of knowledge to accelerate innovation, for the development of solutions, beyond the scope that any organizations or people would do individually. However, collaborative processes are difficult to trigger and remain without policies that ensure a cultural and institutional environment to support SCls. Thus, there is, in fact, the emergence of a type of SCl 4.0 that promotes resilience through technology to face the new challenges of the so-called fourth industrial revolution that produce an era of disruption, expressed by dynamics that are reflected in new technologies, business model, products and strategies in companies and/or start-ups that aim to shake up an industry and change its competitive standards

Keywords: Collaboration. Disruption. Scientific. Technological and Innovation Institution. Technology. Resilience.

\section{INTRODUÇÃO}

A intensidade com que as inovações tecnológicas emergem no século XXI tem propiciado avanços em diferentes tipos de tecnologias, tais como as de informação e comunicação em rede, biotecnologia, neurotecnologia, nanotecnologia, robótica, semicondutores, alimentos e engenharia ambiental, entre outras. Embora essas tecnologias tenham o potencial de gerar transformação positiva e ajudar a abordar as dificuldades em lidar com "grandes desafios societais", a novidade associada à inovação tecnológica também é acompanhada por ansiedades sobre seus impactos ao produzir uma era de disrupções, descrita por novas tecnologias ou startups que visam sacudir uma indústria e alterar seus padrões competitivos.

Levanta-se uma série de questões vitais e complexas que ocorrem a nível mundial, porém, em particular em países emergentes como o Brasil, que vem demonstrando instabilidade institucional e sendo atravessado por frequentes crises políticas, econômicas e sociais que contribuem para a constituição de uma série de situações adversas.

Como uma forma de resposta a esses desafios, a conscientização da sociedade sobre a importância da ciência, tecnologia e inovação, para o desenvolvimento econômico e social do país, tem levado a discussões sobre a efetividade do Sistema Nacional de Ciência Tecnologia e Inovação (SNCTI) e como ele pode ser aprimorado (Cassiolato, Lastres, Maciel Eds., 2003; Viana, 2016; Santa Rita, Radaelli, de Oliveira Sá, Gadelha, de Sousa Junior, Uggioni \& Faiad, 2017; Arraes, 2019).

Entre os diferentes agentes do SNCTI, as Instituições Científicas, Tecnológicas e de Inovação (ICT's) têm obtido maior atenção frente ao seu potencial para auxiliar no aprofundamento das relações entre coletividade científica, empresas, governo e sociedade, por meio da gestão de fluxos de conhecimento técnico especializado, voltados ao aumento da competitividade das empresas, em uma era globalizada (Prest, 2002; Mazzoleni, Nelson, 2007; Åström, Eriksson, Niklasson, Aarnold, 2008; Nath, 2008; CGEE, 2010; Albors $\square$ Garrigos, Zabaleta\&Ganzarain, 2010; Alday\& Salles, 2011; Albors $\square$ Garrigos, Zabaleta, \&Ganzarain, 2010; Giannopoulou, Barlatier\&Pénin, 2019; de Carvalho \& Tonelli, 2020; da Silva Pedro, 2021).

De fato, as ICT's têm procurado aprimorar suas competências na pesquisa e desenvolvimento, formação de recursos humanos, transferência de conhecimento para a sociedade - para o setor empresarial ou para o governo -, além da captação e gestão de recursos financeiros governamentais e privados para a manutenção e desenvolvimento de atividades inovativas, que promovam resiliência por meio da tecnologia para enfrentar os novos desafios da chamada quarta revolução industrial, produtora de uma era de disrupções. Aqui, resiliência é definida como uma meta-capacidade de lidar com situações adversas por meio de estratégias adaptativas, proativas e reativas (Holling, 1973; Coutu, 2002, Sutcliffe \&Vogus, 2003; Hamel \&Valikangas, 2003; Durodie, 2003; McManus, Seville, Brunsden \&Vargo, 2007; Seville, 2009; Pooley \& Cohen, 2010; Bhamra, Dani \& Burnard, 2011; Martinez, Di Nauta, Sarno, 2017; Bergström, van Winsen, Henriqson, 2015; Amir \& Kant, 2018, Blanco, 2018; Barasa, Mbau \& Gilson, 2018; RuizMartin, López-Paredes \& Wainer, 2018; Duchek, 2020); com disrupções expressas por dinâmicas que se refletem em novas tecnologias, modelo de negócios, produtos e estratégias, e em empresas e/ou startups que visam "sacudir" uma indústria e alterar seus padrões competitivos (Christensen, 1997; Christensen e Raynor, 2003; Christensen, McDonald, Altman \& Palmer, 2018; Petzold, Landinez\&Baaken, 2019; Si \& Chen, 2020); Adner \& Lieberman, 2021).

Logo, ao se observar que existe hoje uma pesquisa limitada sobre resiliência e disrupções, em ou por economias emergentes, (Linnenluecke, 2017; Zahra, Wu \& Jeng, 2020) se buscou responder a seguinte questão de pesquisa: como a tecnologia promove resiliência a partir de Instituições Científicas, Tecnológicas e de Inovação (ICT's) no Brasil? Para este fim, se conduziu uma investigação a partir da Aliança Estratégica para a promoção da inovação tecnológica, integrada pela Associação Brasileira das Instituições de Pesquisa Tecnológica e Inovação (Abipti), pela Associação Nacional de Pesquisa, Desenvolvimento e Engenharia das Empresas Inovadoras (Anpei), e pela Associação Nacional de Entidades Promotoras de Empreendimentos Inovadores (Anprotec), que são as principais 
entidades que discutem e representam as ICT's no Brasil. Adicionalmente, se realizou uma análise jurídica das diferentes normas e documentos de referência que regulamentam as atividades de ciência, tecnologia e inovação no ordenamento jurídico brasileiro que diz respeito às Instituições Científicas, Tecnológicas e de Inovação (ICT's), contemplando a Estratégia Nacional de Ciência, Tecnologia e Inovação 2016-2022, a Emenda Constitucional N ${ }^{\circ}$ 85/2015- de 26 de fevereiro de 2015 (EC 85) - e a Lei $n^{\circ} 13.243$ - de 11 de janeiro de 2016 -, sobre estímulos ao desenvolvimento científico, à pesquisa, à capacitação científica e tecnológica e à inovação. Tal Lei também é conhecida como o Marco Legal de CTI, complementada e desenvolvida pelo Decreto $n^{\circ} 9.283$, de 7 de fevereiro de 2018, e veio para alterar a Lei Federal da Inovação, bem como várias normas pré-existentes.

Para responder à questão proposta, o artigo está estruturado da seguinte forma: primeiramente, o texto apresenta teorizações de resiliência e disrupções; na sequência, são apresentados os procedimentos metodológicos adotados na pesquisa; depois são apresentados os resultados da pesquisa em relação às entrevistas, à análise jurídica e às considerações finais - incluindo implicações e limitações deste estudo e as recomendações para futuras pesquisas e-, por fim, o referencial adotado.

\section{REFERENCIAL TEÓRICO}

\subsection{Resiliência, Disrupções e Tecnologia}

O conceito de resiliência é multidisciplinar e multifacetado. Assim sendo, a noção de resiliência está firmemente enraizada na ecologia e as definições de trabalho, usadas por muitos autores, desenvolvidas seguindo a pesquisa original de Holling (1973) relativa à estabilidade dos sistemas socioecológicos e sua capacidade de se recuperarem de crises de longo prazo.

A resiliência, bem conhecida e aplicada em campos de conhecimentos, tais como Gestão, Ecologia, Psicologia, Gestão de Desastres, Organização, Sociologia e Engenharia não possui uma definição amplamente aceita (Bergström, van Winsen, Henriqson, 2015; Amir \& Kant, 2018), tornando-se um tópico de pesquisa emergente nestes diferentes campos e nos quais vários autores já identificaram relações entre eles, propondo várias definições - cada uma ligeiramente alterada dependendo do contexto (Bhamra, Dani \& Burnard, 2011; Linnenluecke, 2017; Ruiz-Martin, López-Paredes \& Wainer, 2018). Entretanto, não existe uma definição inequivocamente aceita de resiliência, mas, no entanto, tal definição constitui-se como uma valiosa abordagem para a pesquisa no campo da gestão (Ruiz-Martin, López-Paredes \& Wainer, 2018; Conz \& Magnani, 2020; Hillmann \& Guenther, 2021).

Resiliência era geralmente considerada como a capacidade de um sistema de continuar a cumprir seus objetivos em face dos desafios. Os conceitos de resiliência costumam enfatizar não apenas a capacidade de um sistema de resistir a choques, mas também de se adaptar e transformar. Horne III e Orr (1998) entendem a resiliência como uma qualidade para responder a mudanças significativas. Assim, a resiliência usualmente é definida como a "capacidade de sobreviver" por meio de uma reconstrução contínua, mantendo estratégias adaptativas, proativas e reativas para lidar com ameaças, riscos, eventos adversos e desafios considerados disruptivos, ou seja, que interrompem o curso normal de um processo (Coutu, 2002, Hamel \&Valikangas, 2003; Sutcliffe \&Vogus, 2003; Durodie, 2003; McManus, Seville, Brunsden \&Vargo, 2007; Seville, 2009; Pooley e Cohen, 2010; Martinez, Di Nauta, Sarno, 2017; Blanco, 2018). A resiliência, então, é influenciada pelos seguintes fatores: recursos materiais, preparação e planejamento, gestão da informação, caminhos colaterais e redundância, processos de governança, práticas de liderança, cultura organizacional, capital humano, redes sociais e colaboração (Barasa, Mbau \& Gilson, 2018). De fato, a resiliência não se refere apenas a um tipo de capacidade organizacional, mas a uma abordagem sobre como as organizações podem gerenciar surpresas e enfrentar ambientes adversos, complexos e incertos, de maneira responsável e proativa, e muitas vezes mesmo antes que as crises ocorram (Tengblad \& Oudhuis, 2018). Dessa forma, inspirados em estudos baseados em processos, a resiliência pode ser definida como uma meta-capacidade composta de três estágios sucessivos: antecipação, enfrentamento e adaptação (Duchek, 2020).

Aqui, resiliência é definida como uma meta-capacidade de lidar com situações adversas por meio de estratégias adaptativas, proativas e reativas (Holling, 1973; Coutu, 2002, Sutcliffe \&Vogus, 2003; Hamel \&Valikangas, 2003; Durodie, 2003; McManus, Seville, Brunsden \&Vargo, 2007; Seville, 2009; Pooley \& Cohen, 2010; Bhamra, Dani \& Burnard, 2011; Martinez, Di Nauta, Sarno, 2017; Bergström, van Winsen, Henriqson, 2015; Amir \& Kant, 2018, Blanco, 2018; Barasa, Mbau \& Gilson, 2018; Ruiz-Martin, López-Paredes \& Wainer, 2018; Duchek, 2020). E nesta direção, segundo ao Projeto Indústria 2027 (IEL, 2017), há uma série de situações adversas que podem afetar o desenvolvimento de setores estratégicos do Brasil, alavancadas por tecnologias como a inteligência artificial, Internet das coisas (em inglês: Internet of Things, loT), produção inteligente e conectada, materiais avançados, nanotecnologia, biotecnologia e armazenamento de energia.

Estas tecnologias, portanto, são reflexos da chamada quarta revolução industrial que, segundo Schwab (2016), transformará fundamentalmente a forma como vivemos, trabalhamos e nos relacionamos, produzindo uma era de disrupções. 
A noção de disrupção ganhou reconhecimento e discussão cada vez maiores de pesquisadores e profissionais após ter sido cunhada originalmente por Christensen (1997) em seu famoso livro The Innovator's Dilemma e explorada a partir de alterações nos processos transacionais de oferta de produtos e serviços, representativos dos modelos de negócio tradicionais, para novos formatos. Tais alterações causam uma disrupção nos processos tradicionais de oferta dos produtos das empresas líderes, permitindo às novas ofertantes um crescimento rápido com eventual deslocamento de suas participações ou compartilhamento de liderança no mercado (Christensen \& Raynor, 2003).

Tem-se suscitado áreas que prometem enriquecer e estender a sua concepção, tais como estratégias de resposta, como as empresas devem responder a disrupções e quais estratégias são eficazes (Christensen, McDonald, Altman \& Palmer, 2018), propondo, assim, que a inovação disruptiva possa ser entendida como ocorrendo por meio de dinâmicas emergentes constituídas a partir: (a) do momento de entrada e dos processos subjacentes que influenciam; (b) da sincronização de eventos e ações e como é moldada pela; (c) adaptabilidade das ações estratégicas (Petzold, Landinez \& Baaken, 2019).

Para Si \& Chen (2020) haveria três perspectivas para a definição de disrupções. A primeira perspectiva para definir os tipos de disrupções é baseada em quatro principais tipos específicos de atividades de inovação: inovação do modelo de negócios; inovação tecnológica; inovação de produto e inovação estratégica. A segunda perspectiva para definir a disrupção é baseada no processo de evolução. E por fim, a terceira perspectiva da definição disrupção é baseada em seu efeito. Entretanto, as definições geradas a partir desta perspectiva são geralmente dispersas e não podem ser sistematicamente categorizadas ou integradas.

Atualmente, a disrupção tem sido definida como "[...] um declínio substancial nas vendas, participação de mercado ou lucratividade de empresas estabelecidas, resultante de ações tomadas por empresas que não são inicialmente rivais diretas das empresas estabelecidas" (Adner \& Lieberman, 2021, p. 92).

Aqui, disrupções são expressas por dinâmicas que se refletem em novas tecnologias, modelo de negócios, produtos e estratégias em empresas e/ou startups que apresentam interesse em movimentar uma indústria, bem como alterar seus padrões competitivos de uma forma diferente de qualquer coisa já experimentada (Christensen, 1997; Christensen e Raynor, 2003; Schwab, 2016; Christensen, McDonald, Altman \& Palmer, 2018; Petzold, Landinez \& Baaken, 2019; Si \& Chen, 2020; Adner \& Lieberman, 2021).

Também chamada de 4.0, a revolução ocorre após três momentos históricos transformadores. O primeiro marcou o ritmo da produção manual à mecanizada, entre 1760 e 1830. O segundo, por volta de 1850, fez uso da eletricidade e das condições que permitiram a manufatura em massa. E o terceiro em meados do século 20 , com o advento da eletrônica, da tecnologia da informação e das telecomunicações. Agora, a mudança que teve início na virada do século XX é baseada na revolução digital, ou "a segunda era da máquina" (Brynjolfsson \& McAfee, 2014). Essa nova fase será impulsionada por uma profusão de novas tecnologias, tais como inteligência artificial, robótica, internet das coisas, veículos autônomos, impressão em 3D, nanotecnologia, biotecnologia, ciência dos materiais, armazenamento de energia e computação quântica, entre outras. Embora essas tecnologias tenham o potencial de gerar transformação positiva e ajudar a abordar as dificuldades em lidar com "grandes desafios societais", a novidade associada à inovação tecnológica também é acompanhada por ansiedades sobre seus impactos possuindo potencial para inibir a resiliência. Para Schwab (2016, p. 35) "a quarta revolução industrial exigirá que atores capacitados reconheçam que são parte de um sistema de poderes distribuídos que requer formas mais colaborativas de interação para que se possa prosperar". Em uma nova reflexão, Schwab (2018) observa que as tecnologias emergentes não são forças predeterminadas fora de controle, nem são ferramentas simples com impactos e consequências conhecidas, já que em seu entendimento é possível demonstrar como cidadãos, líderes empresariais, influenciadores sociais e decisores políticos influenciam a forma como a tecnologia transforma o mundo, moldando um futuro verdadeiramente desejável em um momento de grande incerteza e mudança.

Particularmente, de acordo com a Organização para a Cooperação e Desenvolvimento Econômico (OCDE) de 2020, o Brasil necessita avançar em diversos aspectos para trilhar o caminho da Era Digital: (a) melhorar a conectividade, pois serviços de comunicação de alta qualidade a preços competitivos são cruciais para a transformação digital no Brasil; (b) aumentar a adoção e o uso de tecnologias digitais, pois mesmo tendo feito progressos significativos no que diz respeito à melhoria do acesso à Internet nos últimos anos, o percentual da população adulta que nunca havia usado a Internet no ano de 2018 era de 23\%; (c) aumentar a confiança no ambiente digital, fortalecendo a segurança digital e a proteção aos dados pessoais e aos consumidores; (d) desencadear a inovação digital, ampliando os gastos com Pesquisa e Desenvolvimento (P\&D) no Brasil que, em relação ao Produto Interno Bruto (PIB), que estão acima dos países da América Latina e do Caribe, mas ainda estão atrás dos países da OCDE, Além disso, os gastos empresariais representam uma parcela menor do total de P\&D no Brasil, especialmente no setor de Tecnologias da Informação e Comunicação (TIC), como, por exemplo; (e) promover a transformação digital da economia, além de áreas tais como agricultura, indústria e serviços, com foco em novos modelos de negócios orientados a dados; (f) construir uma abordagem integral do governo (whole-of-government) associada a sua Estratégia de Transformação Digital (E-Digital) para o período de 2018-21, que visa coordenar diferentes iniciativas governamentais quanto a questões digitais.

Nesta direção, a tecnologia desempenha um papel importante no fornecimento de recursos alternativos às pessoas para reconstruir, modificar e desenvolver novas rotinas, padrões de ação, trabalho e socialização frente a uma série de situações adversas (Mark \& Semaan, 2008). Sugere-se que a resiliência, neste contexto, pode ser 
obtida por um tipo peculiar de colaboração que amplia o modelo de produção de conhecimento Triple Helix (Etzkowitz \& Leydesdorff, 2000) para Modelo ou o Quadro do Sistema de Inovação Quadruple / Quintuple Helix, envolvendo: governo, universidade, indústria, sociedade civil e ambiente (Carayannis \& Campbell, 2009; Arnkil et al., 2010; Leydesdorff, 2012; Carayannis \& Campbell, 2012, Carayannis, Barth, \& Campbell, 2012; Carayannis, Grigoroudis, Campbell, Meissner \& Stamati, 2018), reconhecido como CS2FIE2 (no original em língua inglesa: Co-opetitive Spatial and Sectoral Fractal Innovation and Entrepreneurial Ecosystems).

A tríplice hélice pode ser entendida como um modelo para tratar com a inovação, que busca capturar múltiplas e recíprocas relações em diferentes pontos do processo de geração de conhecimento. A primeira dimensão do modelo é a transformação interna em cada uma das hélices, como o desenvolvimento de laços laterais entre empresas, através de alianças estratégicas ou pelo reconhecimento das universidades em assumir também responsabilidade no desenvolvimento econômico. A segunda dimensão importante é a influência que tem uma hélice sobre a outra, ou seja, governo sobre empresa, empresa sobre universidade e assim por diante. A terceira dimensão é a criação de uma nova sobreposição trilateral de redes e organizações, desde a interação entre as três hélices, estabelecida com o propósito de produzir novas ideias e formatos para o desenvolvimento de alta tecnologia (Puffal \& Costa, 2008).

Assim, relações colaborativas entre organizações podem viabilizar o acesso a uma grande quantidade de conhecimento para os processos de inovação (Balestrin, Vargas \& Fayard, 2008, Huizingh, 2011), além de se assemelhar à noção de inovação aberta (Voorberg, Bekkers \& Tummers, 2015).

Para os propósitos deste estudo trata-se de "um paradigma que supõe que as empresas podem e deveriam utilizar ideias externas, assim como ideias internas, e caminhos internos e externos para o mercado, à medida que as empresas buscam avançar suas tecnologias" (Chesbrough, 2003 p. 24). Nisso, se inclui a abertura a vários atores internos e externos que participam de forma colaborativa no processo de inovação, dedicando-se a diferentes tipos de parcerias, aquisição de ideias e recursos do ambiente externo (Chesbrough, 2003). De um modo mais geral, a essência da inovação aberta é a capacidade de criar um ecossistema onde pessoas, organizações e setores possam promover a cocriação (Adner \& Kapoor, 2010; Chesbrough, Kim \& Agogino, 2014; Gawer \& Cusumano, 2014). Isso envolve modelos de negócios - a lógica de criação e captura de valor - que transcendem dinamicamente os limites organizacionais dentro desse ecossistema de inovação (Ritala, Agouridas, Assimakopoulos, \& Gies, 2013; Radziwon, Bogers \& Bilberg, 2017; Holgersson, Granstrand \& Bogers, 2017). Como objeto de pesquisa tem crescido rapidamente desde que o termo foi cunhado (Chesbrough \& Bogers, 2014) e seu futuro é mais extenso, mais colaborativo e mais engajado com uma variedade maior de atores (Chesbrough, 2017) desempenhando um papel fundamental frente às novas tendências tecnológicas que irão impulsionar a inovação (Bogers, Chesbrough \& Moedas, 2018).

Um destes atores que colaboram no processo de inovação trata-se do "intermediário do conhecimento", que assume o papel de conectar outros participantes, podendo ser considerados gestores de uma entidade interorganizacional, voltada ao desenvolvimento de aplicações novas ou significativamente distintas da sua concepção original (Hargadon \& Sutton, 1997; Hibbert, Huxham \& Ring, 2008; Sieget al., 2010). Este intermediário mantém relações com muitos parceiros e desempenha atividades de desenvolvimento de conhecimento específico a partir de sua capacidade de conectar agrupamentos diferentes em sistemas maiores (Long, Cunningham \& Braithwaite, 2013). Além disso, complementa e transforma o aprendizado para incorporá-lo na criação de um novo produto ou processo, em um tipo de suporte, que poderá materializar-se em novos produtos, processos ou modelos de negócios (Hargadon \& Sutton, 1997; Hargadon, 2002; Lingo \& O’Mahony, 2010).

De fato, as referências adotadas suscitam a ideia de que a atuação das ', como intermediários da inovação aberta em um modelo de hélice quádrupla/quíntupla, cria condições para que a tecnologia promova resiliência no Brasil, em particular frente a disrupções decorrentes da chamada quarta revolução industrial.

\section{METODOLOGIA}

A presente pesquisa pode ser classificada como qualitativa (Creswell, 2010) devido sua forma de estudar aspectos subjetivos, além de ser capaz de responder questões que girem em torno do tópico "como" (Yin, 2014). Concentrou-se em um único estudo de caso com múltiplas unidades de análise, com base na Aliança Estratégica para a Promoção da Inovação Tecnológica, por ser integrada pela Associação Brasileira das Instituições de Pesquisa Tecnológica e Inovação (Abipti), pela Associação Nacional de Pesquisa, Desenvolvimento e Engenharia das Empresas Inovadoras (Anpei) e pela Associação Nacional de Entidades Promotoras de Empreendimentos Inovadores (Anprotec), que são as principais entidades que discutem e representam as ICT's no Brasil. Tais entidades são apoiadas por uma análise jurídica das diferentes normas e documentos de referência que regulamentam as atividades de ciência, tecnologia e inovação, no ordenamento jurídico brasileiro, e que dizem respeito às ICT's, contemplando a Estratégia Nacional de Ciência, Tecnologia e Inovação 2016-2022, a Emenda Constitucional № 85/2015 - de 26 de fevereiro de 2015 (EC 85) - e a Lei $n^{\circ} 13.243$ - de 11 de janeiro de 2016 -, sobre estímulos ao desenvolvimento científico, à pesquisa, à capacitação científica e tecnológica e à inovação, conhecida como o Marco Legal de CTI, que foi complementada e desenvolvida pelo Decreto $n^{\circ}$ 9.283, de 7 de fevereiro de 2018 e que veio para alterar a Lei Federal da Inovação, bem como várias normas pré-existentes. 
Tabela 1 - Entidades representativas pesquisadas

\begin{tabular}{ll}
\hline \multicolumn{1}{c}{ Entidade associativa } & \multicolumn{1}{c}{ Descrição } \\
\hline $\begin{array}{l}\text { Associação Brasileira das Instituições de } \\
\text { Pesquisa Tecnológica e Inovação (Abipti). }\end{array}$ & $\begin{array}{l}\text { Representação e promoção da participação das Entidades Públicas e Privadas de } \\
\text { Pesquisa, Desenvolvimento e Inovação Tecnológica (EPDI's) brasileiras associadas } \\
\text { na criação e implementação de políticas voltadas para o Sistema Nacional de Ciência, } \\
\text { Tecnologia e Inovação (SNCTI). }\end{array}$ \\
$\begin{array}{ll}\text { Associação Nacional de Pesquisa } \\
\text { e Desenvolvimento das Empresas } \\
\text { Inovadoras (Anpei). }\end{array}$ & $\begin{array}{l}\text { Representação do segmento das empresas e instituições inovadoras que atuam junto com } \\
\text { as instâncias de governo, os setores produtivos e os formadores de opinião, disseminando } \\
\text { a importância da inovação tecnológica para a competitividade das empresas e o } \\
\text { desenvolvimento do Brasil. }\end{array}$
\end{tabular}

Associação Nacional de Entidades Promotoras de Empreendimentos Inovadores (Anprotec).

Conselho Nacional das Fundações de Apoio às Instituições de Ensino Superior e de Pesquisa Científica e Tecnológica (Confies).

Associação Brasileira dos Reitores das Universidades Estaduais e Municipais (Abruem).

Associação Nacional dos Dirigentes das Instituições Federais de Ensino Superior (Andifes).

Associação Fórum Nacional de Gestores de Inovação e Transferência de Tecnologia (Fortec).
Representação dos interesses das incubadoras de empresas, parques tecnológicos e empreendimentos inovadores no Brasil. Ela atua por meio da promoção de atividades de capacitação, articulação de políticas públicas e geração e disseminação de conhecimentos.

Representação que visa promover o aprimoramento e a troca de experiências entre as fundações associadas, bem como defender seus direitos e prerrogativas comuns.
Representação do sistema estadual e municipal de Educação Superior, que busca aprofundar permanentemente as discussões de temas prioritários para a agenda do Ensino Superior, sempre almejando a harmonia entre ensino, pesquisa, extensão, inovação e internacionalização.

Representação das universidades federais na interlocução com o governo federal, com as associações de professores, de técnico-administrativos, de estudantes e com a sociedade em geral.

Associação civil de direito privado sem fins lucrativos com duração indeterminada, de representação dos responsáveis nas universidades, institutos de pesquisa, instituições gestoras de inovação e pessoas físicas, pelo gerenciamento das políticas de inovação e das atividades relacionadas à propriedade intelectual e à transferência de tecnologia incluindo-se, neste conceito, os Núcleos de Inovação Tecnológica (NIT's), agências, escritórios e congêneres.

Fonte: Elaborada pelos autores

Dessa forma, a coleta de dados ocorreu em duas etapas. A primeira de reconhecimento e exploração, em que se buscou entender as principais características das ICT's no Brasil (ambiente institucional, gestão, papel, metodologias adotadas, e colaboração) com um grupo de entidades representativas de comunidades científicas, tecnológicas e de inovação, acadêmicas e do ensino superior. Nessa fase as entrevistas foram realizadas pessoalmente, por Skype ou por telefone, com duração média de uma hora, além de terem sido registradas com equipamento de gravação de áudio. A seleção levou em conta dois critérios: (1) a sua representatividade e (2) a disponibilidade para integrar a pesquisa.

As entidades representativas (Tabela1) estudadas foram: Associação Brasileira das Instituições de Pesquisa Tecnológica e Inovação (Abipti), Associação Nacional de Pesquisa e Desenvolvimento das Empresas Inovadoras (Anpei), Associação Nacional de Entidades Promotoras de Empreendimentos Inovadores (Anprotec), Conselho Nacional das Fundações de Apoio às Instituições de Ensino Superior e de Pesquisa Científica e Tecnológica (Confies), Conselho Nacional de Secretários para Assuntos de Ciência Tecnologia e Inovação (Consecti), Associação Brasileira dos Reitores das Universidades Estaduais e Municipais (Abruem), Associação Nacional dos Dirigentes das Instituições Federais de Ensino Superior (Andifes), Associação Fórum Nacional de Gestores de Inovação e Transferência de Tecnologia (Fortec).

A segunda etapa de investigação se buscou compreender como a tecnologia promove resiliência a partir de Instituições Científicas, Tecnológicas e de Inovação (ICT's) no Brasil. Foi adotado um roteiro semiestruturado com estratégias adaptativas, proativas e reativas adotadas pelas ICT's, de acordo com as percepções das entidades representativas que integram a Aliança Estratégica para a Promoção da Inovação Tecnológica. AAliança Estratégica para a Promoção da Inovação Tecnológica, integrada pela Abipti, pela Anpei e pela Anprotec, consiste em uma articulação institucional e um intercâmbio permanente de informações entre as entidades, ampliando o espaço da pesquisa tecnológica no âmbito das políticas públicas nacionais.

As entrevistas foram realizadas pessoalmente, por Skype ou por telefone, com duração média de duas horas e registradas com equipamento de gravação de áudio. A seleção levou em conta dois critérios: (1) seu vínculo com a dinâmica das ICT's e (2) seus esforços de colaboração mútua. 
Foi adotado método de triangulação (Flick, 2012), combinando distintos dados, provenientes de várias fontes de evidências e sob uma abordagem teórica, sem a utilização de métodos distintos, produzindo-se, assim, um conhecimento adicional em relação ao que seria possível, caso fosse adotada uma única perspectiva. Neste caso, os dados foram coletados em momentos diferentes, e com pessoas diferentes, mesmo nas três entidades representativas que integram a Aliança Estratégica para a Promoção da inovação Tecnológica. Ou seja, entrevistaram-se gestores ou secretários-executivos na fase exploratória, enquanto que na fase de investigação o foco foi nos presidentes das entidades. Além disso, foram coletados dados em seus websites, estatutos e materiais publicitários como cards, flyers e cartilhas. Dados também foram coletados das normas e documentos de referência que regulamentam as atividades de ciência, tecnologia e inovação no ordenamento jurídico brasileiro, que dizem respeito às ICT's e que foram elencados a partir das entrevistas.

A triangulação de dados no processo de análise deu subsídio maior para a construção das categorias ao possibilitar a confirmação destas a partir de temáticas que se repetiam e se confirmavam ao longo das técnicas de produção de dados. No entanto, mais do que convergências, percebeu-se a presença da complementaridade dos dados, já que as temáticas apresentaram-se mais concisas ao longo das técnicas.

Para análise de dados, a técnica de análise de conteúdo foi adotada para inferir conhecimento por meio da geração de indicadores quantitativos (Bardin, 2011). As análises seguiram três fases: a pré-análise, a exploração do material oriundo da coleta de dados e o tratamento da informação e sua interpretação. Na fase de pré-análise se conduziu a coleta dos aspectos gerais e características das IC'Ts. Na fase de exploração do material, as informações brutas oriundas da coleta de dados foram espelhadas na literatura de referência. Já na fase de tratamento da informação e sua interpretação, as informações obtidas foram compiladas em um único quadro em que se procurou expressar como a tecnologia promove resiliência, a partir de Instituições Científicas, Tecnológicas e de Inovação (ICT's) no Brasil.

Por fim, os dados foram constantemente comparados (Strauss \& Corbin, 1990) entre a teoria e os resultados, para promover a discussão sobre como a tecnologia promove resiliência, a partir de ICT's no Brasil.

\section{RESULTADOS}

\subsection{ICT's no Ordenamento Jurídico Brasileiro}

O ambiente institucional das ICT's está caracterizado pela sua inserção no terceiro nível de atores do Sistema Nacional de Ciência, Tecnologia e Inovação (SNCTI) (Figura 1). Já os operadores de Ciência, Tecnologia e Inovação (CT\&l), onde são geradas as inovações, desenvolvidas as tecnologias e realizadas as pesquisas, foram objeto de diretrizes no nível político e de alocações de recursos no estágio das Agências de Fomento.

Enquanto nos outros dois níveis as atividades são desempenhadas majoritariamente por gestores do Sistema, neste nível as iniciativas contam com o trabalho de pesquisadores e tecnologistas. Universidades podem ser tomadas como ICT's, devendo-se considerar também sob essa nomenclatura outros operadores relevantes tais como: as Instituições de Pesquisa Tecnológica e Inovação (privadas); os Institutos Federais de Educação, Ciência e Tecnologia (IFE); e os Institutos Estaduais de CT\&I. Nesse nível, cabe destacar o papel exercido pelos Institutos Nacionais de Ciência e Tecnologia (INCT's) que congregam as unidades de pesquisa de maior excelência no País e tem por objetivo desenvolver a pesquisa e criar patentes para o país.

Um marco importante na CT\&I brasileira que contribuiu para a colaboração entre as ICT's e outros atores do SNCTI foi a institucionalização, através da Emenda Constitucional n ${ }^{\circ}$ 85, em 26 de fevereiro de 2015, do Sistema Nacional de Ciência, Tecnologia e Inovação (SNCTI), que passou a ser organizado em regime de colaboração entre entes, tanto públicos quanto privados, com vistas ao desenvolvimento científico e tecnológico do país. Entretanto, questiona-se como se poderia identificar e promover a facilitação da interação entre atores (organizações e pessoas) de áreas envolvidas no desenvolvimento e financiamento de processos e produtos inovadores, sem pensar na formatação de estruturas verticais (hierarquias exclusivamente burocráticas) e, assim, abrindo caminho para interações horizontais (colaboração) (CGEE, 2016). Notadamente, uma das conclusões da auditoria operacional realizada pelo Tribunal de Contas da União do Ministério da Ciência, Tecnologia e Inovações (MCTI) e outros órgãos e entidades federais expressa no Acordão TCU 1237/2019 foi a ausência de uma estrutura de coordenação das políticas federais de fomento à inovação (Arraes, 2019).

Nesta direção, o aprimoramento do arcabouço legal e institucional para essa modalidade de colaboração em Pesquisa, Desenvolvimento e Inovação (PD\&l), entre academia e indústria, é um dos principais desafios enfrentados para ICT's no Brasil, mas que segue em refinamento a partir da regulamentação do Decreto n. ${ }^{\circ} 9.283$, de 2018, alterando a Lei de Inovação (Lei $n^{\circ} 10.973$, de 2004, já modificada pela Lei $n^{\circ} 13.243$, de 2016), Lei das Licitações (Art. 24, $\S 3^{\circ}$, e o Art. 32, $\S 7^{\circ}$, da Lei $n^{\circ} 8.666$, de 1993), o Art. $1^{\circ}$ da Lei $n^{\circ} 8.010$, de 1990, e o Art. $2^{\circ}$, caput, inciso I, alínea "g", da Lei no 8.032, de 1990 e do Projeto de Lei do Senado (PLS) 226/2016 no Congresso, que visa recompor oito itens vetados. Com isso, há um entendimento de que os vetos presidenciais apontados impedem que a Lei opere na plenitude de sua concepção, desconsiderando propostas de atuação que estruturam o SNCTI para operar de acordo com o potencial do país. 
Figura 1 - Principais atores do SNCTI
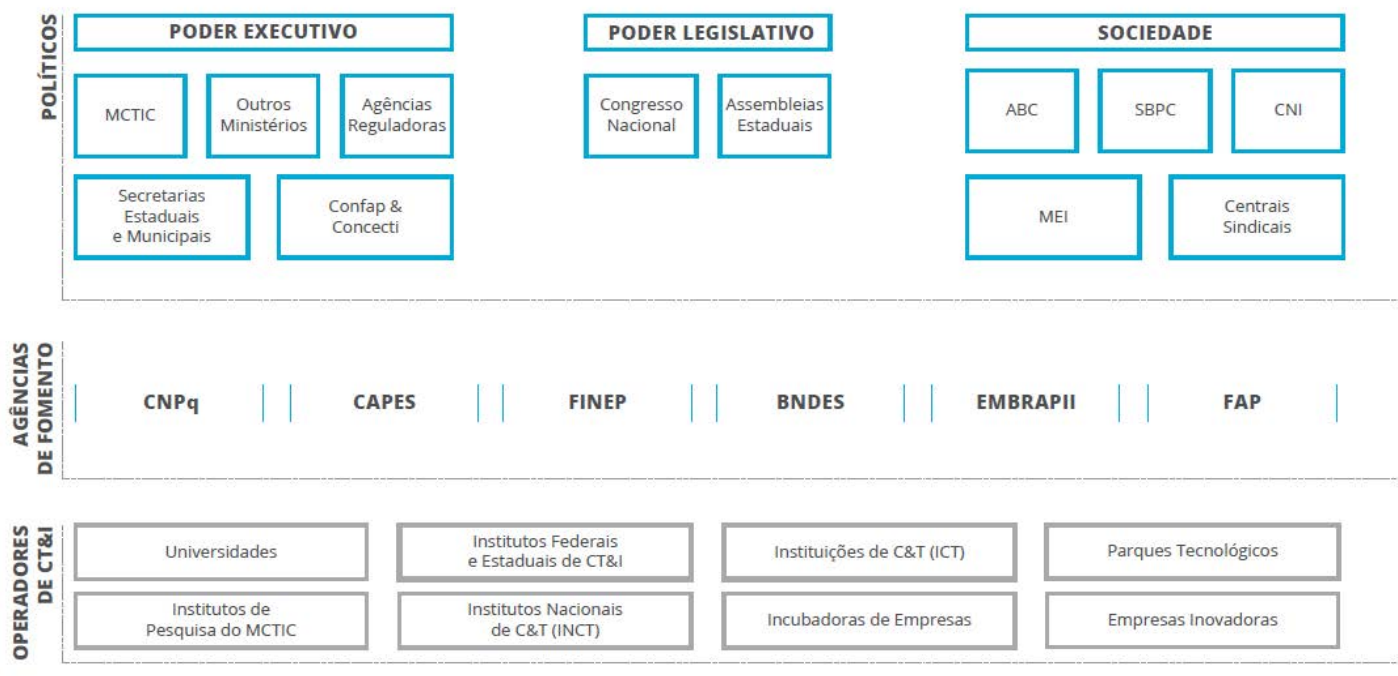

Fonte: ENCTI 2016-2022 (Brasil, 2016).

Particularmente, o conceito de ICT foi estabelecido na Lei de Inovação (Brasil, 2004, Artigo 2o, inciso V) com o intuito de identificar o agente principal desse regramento jurídico. Assim, a figura das ICT's abrangia, em sua redação original, os entes da administração pública que executam atividades de pesquisa e inovação, tais como universidades e institutos de pesquisa públicos. No entanto, o SNCTI é reconhecidamente muito mais amplo, abrangendo instituições de personalidades jurídicas diversas e vinculadas à esfera pública - que não são exclusivamente os órgãos ou as entidades da administração pública - e que também realizam atividades de pesquisa e inovação, tais como as entidades empresariais - a exemplo de empresas públicas, como a Empresa Brasileira de Pesquisa Agropecuária (Embrapa), ou as sociedades de economias mistas, como a Petrobras e entidades sem fins lucrativos. Diante disso, ocorreu a expansão do conceito de ICT a outras entidades, como as instituições de direito privado sem fins lucrativos, abrangendo e oficializando importantes instituições que já fazem parte do SNI e que produzem pesquisa e geram inovações de grande impacto nacional, como as organizações sociais.

Neste sentido, deve ser ressaltada a criação, no segundo nível de atores do SNCTI (Figura 1), as agências de fomento da Empresa Brasileira de Pesquisa e Inovação Industrial (Embrapii) e Organização Social, cuja missão é apoiar projetos empresariais que tenham como base a inovação por meio da cooperação universidade-empresa, utilizando-se de mecanismos ágeis e transparentes de contratação.

As origens das ICT's remontam ao processo de capacitação institucional nas áreas de Tecnologia Industrial Básica - TIB -, em que o Governo Brasileiro concebeu, entre 1982 e 1984, o Subprograma de Tecnologia Industrial Básica dentro do Programa de Apoio ao Desenvolvimento Científico e Tecnológico (PADCT), executado mediante três sucessivos acordos de empréstimo com o Banco Mundial até 1998. Na sequência e, em decorrência das TIB, ocorreu a qualificação de laboratórios e acreditação pelo Instituto Nacional de Metrologia, Qualidade e Tecnologia (Inmetro), os quais fornecem serviços diretamente às indústrias. Sequencialmente, houve o momento das plantas piloto (plantas de processo em pequena escala) que permitiriam determinar se um determinado processo é tecnicamente e economicamente viável, bem como estabelecer os parâmetros operacionais do referido processo para a subsequente concepção e construção da planta em escala industrial. E, nos dias de hoje, voltam-se a simulações que permitem o teste e otimização de processos e produtos ainda na fase de concepção, diminuindo os custos e o tempo de criação.

Ainda sobre esse assunto, o Decreto n. ${ }^{\circ}$ 9.283/2018 criou mecanismos para integrar ICT's e incentivar investimentos em pesquisa, como por exemplo: simplificar a celebração de convênios para a promoção da pesquisa pública e procedimentos de importação de bens e insumos para pesquisa; a internacionalização de instituições científicas e tecnológicas para aumentar a interação ICT's e as empresas; incrementar a promoção de ecossistemas de inovação; diversificar instrumentos financeiros de apoio à inovação, permitir maior compartilhamento de recursos entre entes públicos e privados; gerar novos estímulos para a realização de encomendas tecnológicas e flexibilidade no remanejamento entre recursos orçamentários.

Com o objetivo de avançar na promoção de um ambiente regulatório mais seguro e estimulante para a inovação no Brasil, grande peso é dado ao estabelecimento de mecanismos de estímulo à participação de ICT's em atividades de inovação associadas ao segmento produtivo. ICT é definida no Decreto n. ${ }^{\circ} 9.283$, de 2018 , em seu Art. $2^{\circ}$, Inciso IV, como "órgão ou entidade da administração pública, direta ou indireta, ou pessoa jurídica de direito privado sem fins lucrativos, legalmente constituída sob as leis brasileiras, com sede e foro no país, que inclua em sua missão 
institucional os objetivos social ou estatutário, entre outros, a pesquisa básica ou aplicada de caráter científico ou tecnológico, ou o desenvolvimento de novos produtos, serviços ou processos" (BRASIL, 2018).

Dos regulamentos estabelecidos, merece atenção o que trata da instituição da política de inovação nas ICT's de direito público, que dispõe sobre: a organização e a gestão dos processos que orientarão a transferência de tecnologia e a geração de inovação no ambiente produtivo. Mesmo não sendo obrigatório, é conveniente, também, às ICT's privadas estabelecer sua política de inovação, para consolidar sua capacidade de contribuir para o SNCTI e alavancar recursos advindos de políticas públicas nacionais de fomento às atividades de CT\&I.

A publicação do Decreto 10.534/2020 instituiu a Política Nacional de Inovação (PNI) como resposta do governo às avaliações e às recomendações do Acordão TCU 1237/2019. Porém, apesar da celeridade em sua formulação, não respondeu satisfatoriamente às falhas de coordenação horizontal e vertical do SNCTI e nem supre a recomendação de definição de prioridades. Para da Silva Pedro (2021), apesar de haver a necessidade de mais tempo para articular no Congresso um Projeto de Lei, teria sido mais coerente e robusto ter seguido o caminho apontado pelo Artigo 219B, da Constituição para instituir a Política e o Sistema Nacional de CTI via Lei Complementar. Sem contar que, para além do apontamento das prioridades como elemento-chave de coordenação do SNCTI e das políticas de inovação das ICT's, entende-se que essas prioridades seriam fundamentais para o alinhamento e o acoplamento a serem construídos com a Estratégia Nacional de Propriedade Intelectual (Enpi) (da Silva Pedro, 2021). A definição clara de áreas e de setores tecnológicos a serem priorizados para a inovação nos sistemas produtivos e de serviços otimizaria, assim, a criação, a apropriação e a difusão de inovações, bem como balizaria o alinhamento da PNI com a Política de Desenvolvimento Regional (PNDR) e as estratégias de especialização inteligente de regiões e de territórios construindo bases para direcionar a política de inovação das ICT's e outros planos e estratégias específicas, como, por exemplo, o Plano Nacional de Internet das Coisas e a Estratégia Brasileira de Inteligência Artificial.

De maneira geral as ICT's são percebidas como organizações públicas ou privadas e seu papel está voltado às atividades de pesquisa de caráter científico ou tecnológico, que através da transferência do conhecimento podem contribuir para a inovação nas empresas. Ou seja, seu propósito e legado de criação reside na transformação deste conhecimento em riqueza. Dessa forma, sua gestão é condicionada ao "marco legal da inovação", regulamentado pelo Decreto $n^{\circ} 9.283 / 2018$ e por outros dispositivos específicos, como no caso das ICT's públicas, que devem se adequar às normas da Lei 13.303/2016, também chamada de Lei das Estatais, cujas são estruturadas em duas partes. Na primeira, há um conjunto de normas sobre governança corporativa, transparência na gestão e mecanismos de controle da atividade empresarial. Na segunda, regramento sobre licitação e contratação a serem observadas pelas empresas estatais.

As metodologias adotadas pelas ICT's são pautadas, prioritariamente, por normas e padrões de referências, dos quais se pode destacar: (i) norma técnica internacional sobre competência de laboratórios de ensaio e calibração, a ISO/ IEC 17025; (ii) norma ABNT NBR 16501:2011 - Diretrizes para Sistemas de Gestão da Pesquisa, do Desenvolvimento e da Inovação. Ainda sobre essas metodologias temos o reconhecimento das ICT's como Unidades Embrapii, as quais adotam o Sistema de Excelência Operacional Embrapii e o seu manual de operações que apresenta a descrição dos requisitos operacionais para uma Unidade Embrapii (UE); e a adoção de boas práticas oriundas do Programa de Excelência na Gestão da Abipti, que tinha por objetivo a melhoria contínua das práticas de gestão das entidades de pesquisa, desenvolvimento e inovação (EPDl's) no país para orientá-las a tornarem-se mais competitivas. A partir disso, e adicionalmente, aponta-se a série de normas ISO 56000 em construção sob a responsabilidade da Organização Internacional de Normalização (ISO, na sigla em inglês, por meio do comitê técnico, para tratar do tema o ISO/TC 279, do qual o Brasil faz parte com a Comissão de Estudo Especial de Gestão da Pesquisa, Desenvolvimento e Inovação (CEE-130), da Associação Brasileira de Normas Técnicas (ABNT).

As parcerias público-privadas (PPP's), os centros de pesquisa conjuntos, os licenciamentos de propriedade intelectual, além de incentivos para a mobilidade de acadêmicos empreendedores, estão entre os principais mecanismos que viabilizam a colaboração. Além dos esforços governamentais, outros mecanismos estão ganhando importância como os financiamentos baseados em propriedade Intelectual (securitização), o financiamento coletivo (crowdfunding) e financiamentos à pesquisa voltados para a colaboração internacional. Adicionalmente, a colaboração com Estados, Municípios e instituições de ensino e pesquisa parecem ser alternativas significativas para o fortalecimento do SNCTI. Além disso, recentemente houve a oficialização de um novo tipo de colaboração inédito no Brasil entre entidades voltadas à inovação por meio da criação da Rede Nacional de Associações de Inovação (RNAll). Tratase de uma iniciativa inédita de colaboração das grandes entidades fomentadoras do empreendedorismo inovador, em prol de todo o ecossistema brasileiro de ciência, tecnologia e inovação. É composta pela Abipti (Associação Brasileira das Instituições de Pesquisa Tecnológica e Inovação); ABstartup (Associação Brasileira de Startups); Abvcap (Associação Brasileira de Private Equity\& Venture Capital); Anjos do Brasil; Anpei (Associação Nacional de Pesquisa e Desenvolvimento das Empresas Inovadoras); Anprotec (Associação Nacional de Entidades Promotoras de Empreendimentos Inovadores) e pelo Fortec (Fórum Nacional de Gestores de Inovação e Transferência de Tecnologia).

Juntas, essas associações representam mais de mais de 150 instituições de pesquisa, 300 gestores de núcleos de inovação, 370 ambientes de inovação (incubadoras, aceleradoras, parques tecnológicos), 120 investidores de venture capital, 5000 startups, 7000 investidores anjos, e 200 empresas inovadoras de grande e médio porte, na colaboração e fortalecimento de ações, públicas e privadas, focadas na transformação do conhecimento em riqueza. 


\section{DISCUSSÃO}

Os dados coletados demonstram que a tecnologia promove resiliência a partir de ICT's frente às disrupções decorrentes da chamada quarta revolução industrial.

A associação entre as estratégias adaptativas, proativas e reativas que traduzem o conceito de resiliência, e os quatro processos transformadores (produção manual à mecanizada; uso da eletricidade e manufatura em massa; eletrônica e Tecnologias da Informação e Comunicação ( $T / C$ 's), das condições que permitiram a manufatura em massa, além dos sistemas que foram construídos sobre a infraestrutura da revolução anterior, denominada de quarta revolução industrial) permitem interpretar os principais momentos da evolução das ICT's brasileiras (capacitação institucional nas áreas de TIB, fornecimento de serviços diretamente às indústrias, plantas piloto e simulações) para caracterizar as ICT's dos dias de hoje como ICT's 4.0, fazendo alusão ao momento das simulações.

Dessa forma, tal entendimento pode ser percebido na Figura 1.

Figura 2 - Promoção da resiliência por meio da tecnologia nas ICT's no Brasil.

Erocessos
transformadores
$\begin{aligned} & \text { Produção manual à } \\ & \text { mecanizada. }\end{aligned}$
$\begin{aligned} & \text { Eletricidade e manufatura } \\ & \text { em massa. }\end{aligned}$
$\begin{aligned} & \text { Eletrônica, tecnologia da } \\ & \text { informação e } \\ & \text { telecomunicaçães. }\end{aligned}$
$\begin{aligned} & \text { Sistemas que foram } \\ & \text { construídos sobre a } \\ & \text { infraestrutura da revolução. }\end{aligned}$

As cores representam o nível de atendimento às demandas dos quatro processos transformadores: verde $=$ atendido, amarelo $=$ parcialmente atendido, e vermelho $=$ não atendido. $O$ sentido das setas representa o nível de atenção dispensada ou a ser dispensada: vertical para baixo = esgotamento, horizontal = manutenção, diagonal para cima $=$ incremento, diagonal para baixo $=$ declinio, e vertical para cima $=$ emergência .

A noção de emergência apresentada tem duas interpretações complementares. Uma que diz respeito ao ato ou efeito de emergir, fazendo referência às disrupções decorrentes da chamada "quarta revolução industrial", particularmente marcada pela convergência de tecnologias digitais. Outra que sugere uma situação grave, perigosa, momento crítico ou fortuito que procura representar uma era de disrupções.

Para isso deve haver condições que permitam a sua operação no modelo de hélice quadrupla/quíntupla onde o governo, empresas, academia, sociedade e ambiente possam se conectar a partir das ICT's para desenvolver soluções, colaborativamente. Entretanto, os processos colaborativos são difíceis de desencadear e manter sem políticas que assegurem um ambiente cultural e institucional de apoio às ICT's. Isso se dá devido a interpretação de que as instituições privadas, particularmente as Instituições de Pesquisa Tecnológica e Inovação, levariam certa vantagem frente as públicas, devido sua flexibilidade e compatibilidade com as demandas do mercado e, especialmente, aquelas com familiaridade com as TIC's diante das disrupções decorrentes da chamada quarta revolução industrial, e particularmente marcada pela convergência de tecnologias digitais, que permite ao Brasil avançar em diversos aspectos para trilhar o caminho da Era Digital.

\section{CONSIDERAÇÕES FINAIS}

Este trabalho teórico-empírico buscou examinar como a tecnologia promove resiliência, a partir de Instituições Científicas, Tecnológicas e de Inovação (ICT's) no Brasil. Para este fim, foi adotada uma abordagem 
qualitativa e concentrou-se em um único estudo de caso com múltiplas unidades de análise, com base na Aliança Estratégica para a Promoção da Inovação Tecnológica, integrada pela Abipti, pela Anpei e pela Anprotec, apoiada por uma análise jurídica das diferentes normas e documentos de referência que regulamentam as atividades de CT\&I no ordenamento jurídico brasileiro que dizem respeito às ICT's.

As evidências obtidas demonstram que há, de fato, um tipo de ICT 4.0 em que a tecnologia promove resiliência para enfrentar os novos desafios da chamada quarta revolução industrial que produzem uma era de disrupções, por meio da adoção de estratégias adaptativas, proativas e reativas, interpretadas de acordo com os principais momentos da evolução das ICT's brasileiras. Além disso, geram implicações para gestores, formuladores de políticas públicas e acadêmicos em diferentes campos de conhecimento.

Gestores de empresas podem fazer uso dos achados obtidos para criar e/ou aprimorar fluxos de entrada e saída de conhecimento para acelerar a inovação em conexão com as ICT's. Gestores de ICT's podem se beneficiar deste estudo como uma entrada em seu processo de planejamento, permitindo alinhar suas ações a partir da adoção das estratégias resilientes - reativas, adaptativas e proativas - e diante dos sistemas construídos sobre a infraestrutura da revolução anterior, denominada de quarta revolução industrial, além das estratégias proativas relativas para eletrônica e TIC's.

Aos formuladores de políticas, a ampliação da compreensão sobre como a tecnologia promove resiliência, a partir de ICT's no Brasil, permite a obtenção de novos discernimentos a serem incorporados em normas e documentos de referência que regulamentam as atividades de CT\&I no ordenamento jurídico brasileiro que dizem respeito às ICT's. Podem envolver a criação das bases necessárias para o desenvolvimento de processos colaborativos que assegurem um ambiente cultural e institucional de apoio às ICT's; bem como a promoção daquelas que demonstrem flexibilidade e compatibilidade com as demandas do mercado e, especialmente, aquelas com familiaridade com as TIC's, diante das disrupções decorrentes da chamada quarta revolução industrial, particularmente marcada pela convergência de tecnologias digitais aqui percebidas como as Instituições de Pesquisa Tecnológica e Inovação privada.

Para acadêmicos, são gerados novos discernimentos sobre a resiliência, como um fenômeno multidisciplinar, que nesta pesquisa explora um contexto em que a tecnologia fundamenta a adoção de estratégias resilientes para enfrentamento de novos desafios da chamada quarta revolução industrial, que produzem uma era de disrupções.

Ainda, aponta para futuras pesquisas teóricas e empíricas resultantes deste encontro. Tais pesquisas teóricas poderiam ampliar o entendimento sobre as relações resiliência-tecnologia, resiliência-disrupções, tecnologia-disrupções ou ampliar a múltipla combinação de resiliência-tecnologia-disrupções abordadas neste estudo. Além disso, a compreensão destas dimensões na formulação e avaliação de políticas públicas para a inovação teria o potencial de trazer diversas contribuições que transbordariam a academia. Pesquisas empíricas explorariam o entendimento sobre ICT's e seu papel no SNCTI, suas formas de colaboração por meio de entidades representativas ou redes, como, por exemplo, a Abipti e RNAII, além de particularidades das ICT's e seus modelos de operação, como, por exemplo, as Instituições de Pesquisa Tecnológica e Inovação, sejam elas privadas ou públicas, com ênfase em TIC's ou outras competências. Por fim, estudos prospectivos poderiam delinear o futuro das ICT's no Brasil como ICT's 4.0.

Porém, como limitação desta pesquisa, considera-se que a extensão da literatura que explora temas como políticas públicas e novas tecnologias, ou mesmo inovações, poderiam ampliar a compreensão contextual do leitor. Além disso, em função do método qualitativo adotado, os achados obtidos não permitem sua generalização.

\section{REFERÊNCIAS}

ADNER, R.; KAPOOR, R. Value creation in innovationeco systems: how the structure of technological interdependence affects firm performance in new technology generations. Strategic Management Journal,[S. I.], v. 31, n. 3, p. 306333, 2010.

ADNER, R.; LIEBERMAN, M. Disruption through complements. Strategy Science,[S. I.],v.6, n. 1, p. 91-109, 2021.

ALBORS-GARRIGÓS, J.; RINCON-DIAZ, C. A.; IGARTUA-LOPEZ, J. I. Research technology organisations as leaders of R\&D collaboration with SMEs: role, barriers and facilitators. Technology Analysis \& Strategic Management, [S. I.], v. 26, n. 1, p. 37-53, 2014.

AMIR, S.; KANT, V. Sociotechnical Resilience: A Preliminary Concept. RiskAnalysis, v. 38, p. 8-16. 2018.

ARNKIL, R.; JÄRVENSIVU, A.; KOSKI, P.; PIIRAINEN, T. Exploring the Quadruple Helix Report of Quadruple Helix Research For the CLIQ Project. [S. I.: s. n.], 2010.

ARRAES, A. Relatório de auditoria operacional. TC 017.220/2018-1. Disponível em: Link. Acesso em: 8 maio 2021.

ASTRÖM, T.; ERIKSSON, Marie-Louise; ARNOLD, E. International comparison of five institute systems. Denmark: Forsknings-og Innovationsstyrelsen, Copenhagen, 2008. 
BALESTRIN, A.; VARGAS, L. M.; FAYARD, P. Knowledge creation in small-firm network. Journal of Knowledge Management, [S. I.], v. 12, n. 2 , p. 94-106, 2008.

BARASA, E.; MBAU, R.; GILSON, L. What is resilience and how can it be nurtured? A systematic review of empirical literature on organizational resilience. International journal of health policy and management, v. 7, n. 6, p. 491, 2018.

BARDIN, L. Análise de conteúdo. São Paulo: Edições 70, 2011.

BERGSTRÖM, J.; VAN WINSEN, R.; HENRIQSON, E. On the rationale of resilience in the domain of safety: A literature review. Reliability Engineering \& System Safety, v. 141, p. 131-141, 2015.

BHAMRA, R.; DANI, S.; BURNARD, K. Resilience: the concept, a literature review and future directions. International Journal of Production Research, [S. I.], v. 49, n. 18, p. 5375-5393, 2011.

BLANCO, J. M. M. Organizational Resilience. How Learning Sustains Organizations in Crisis, Disaster, and Breakdown by D. Christopher Kayes. The Learning Organization. [S. I.], v. 25, n. 2, p. 143-146. 2018.

BOGERS, M.; CHESBROUGH, H.; MOEDAS, C. Open innovation: research, practices, and policies. California Management Review, [S. I.], v. 60, n. 2, p. 5-16, 2018.

BRASIL(a). Ministério de Ciência Tecnologia e Inovação. Estratégia nacional de ciência, tecnologia e inovação 2016-2019. Brasília: MCTIC, 2016.

BRASIL(b). Decreto ${ }^{\circ}$ 9.283, de 7 de fevereiro de 2018. Link.

BRYNJOLFSSON, E.; MCAFEE, A. The second machine age: Work, progress, and prosperity in a time of brilliant technologies. New York: WW Norton \& Company, 2014.

CARAYANNIS, E. G.; BARTH, T. D.; CAMPBELL, D. F. J. The Quintuple Helix innovation model: global warming as a challenge and driver for innovation. Journal of innovation and entrepreneurship, [S. I.], v. 1, n. 1, p. 1-12, 2012.

CARAYANNIS, E. G.; CAMPBELL, D. F. Mode 3 knowledge production in quadruple Helix innovation systems. New York: Springer 2012.

CARAYANNIS, E. G.; CAMPBELL, D. F. 'Mode 3'and'Quadruple Helix': toward a 21st century fractal innovation ecosystem. International Journal of Technology Management, [S. I.], v. 46, n. 3-4, p. 201-234., 2009.

CARAYANNIS, E. G.; GRIGOROUDIS, E.; CAMPBELL, D. F.; MEISSNER, D.; STAMATI, D. The ecosystem as helix: an exploratory theory-building study of regional co-opetitive entrepreneurial ecosystems as Quadruple/Quintuple Helix Innovation Models. R\&D Management, [S. I.], v. 48, n. 1, p. 148-162, 2018.

CARVALHO, B. G. de; TONELLI, D. F. Limites e possibilidades do marco legal da CT\&I de 2016 para as instituições científicas e tecnológicas do Brasil. Revista de Administração, Sociedade e Inovação, [S. I.], v. 6, n. 2, p. 6-24, 2020.

CASSIOLATO, J. E.; LASTRES, H.M.M.; MACIEL, M.L. (ed.). Systems of innovation and development: evidence from Brazil. Cheltenham: Edward Elgar. 2003. (New Horizons in theEconomicsoflnnovation Series).

CHESBROUGH, $\mathrm{H}$. The future of open innovation: The future of open innovation is more extensive, more collaborative, and more engaged with a wider variety of participants. Research-Technology Management, [S. I.], v. 60, n. 1, p. 35-38, 2017.

CHESBROUGH, H. W. Open innovation: The new imperative for creating and profiting from technology. Boston: Harvard Business Press, 2003.

CHESBROUGH, H.; BOGERS, M. Explicating open innovation: Clarifying an emerging paradigm for understanding innovation. New Frontiers in Open Innovation. Oxford: Oxford University Press, Forthcoming, p. 3-28, 2014.

CHESBROUGH, H.; KIM, S.; AGOGINO, A. Chez Panisse: Building an open innovation ecosystem. California Management Review, [S. I.], v. 56, n. 4, p.144-171, 2014. 
CHRISTENSEN, C. M. The innovator's dilemma: when new technologies cause great firms to fail. Boston: Harvard Business Review Press, 2013.

CHRISTENSEN, C. M.; McDONALD, R.; ALTMAN, E. J.; PALMER, J. E. Disruptive innovation: An intellectual history and directions for future research. Journal of Management Studies, [S. I.], v. 55, n. 7, p. 1043-1078, 2018.

CHRISTENSEN, C. M.; RAYNOR, M. E. Why hard-nosed executives should care about management theory. Harvard Business Review, [S. I.] v. 81, n. 9, p. 66-75, 2003.

CONZ, E.; MAGNANI, G. A dynamic perspective on the resilience of firms: A systematic literature review and a framework for future research. European Management Journal, [S. I.], v. 38, n. 3, p. 400-412, 2020.

COUTU, D. L. How resilience works. Harvard Business Review, [S. I.], v. 80, n. 5, p. 46-56, 2002.

CRESWELL, J. W. W. Projeto de pesquisa: métodos qualitativo, quantitativo e misto. 2. ed. Porto Alegre: Bookman, 2010.

DUCHEK, S. Organizational resilience: a capability-based conceptualization. Business Research, [S. I.], v. 13, n. 1, p. 215-246, 2020.

DURODIE, B. Is real resilience attainable?. Homeland Security \& Resilience Monitor, [S. I.], v. 2 n. 6, p. 15-19, 2003.

ETZKOWITZ, H.; LEYDESDORFF, L. The dynamics of innovation: from National Systems and "Mode 2" to a Triple Helix of university-industry-government relations. Research Policy, [S. I.], v. 29, n. 2, p. 109-123, 2000.

FLICK, U. Introdução à metodologia de pesquisa: um guia para iniciantes. Porto Alegre: Penso, 2012.

GAWER, A.; CUSUMANO, M. A. Industry platforms and ecosystem innovation. Journal of Product Innovation Management, [S. I.], v. 31,n. 3, p. 417-433, 2014.

GEORGHIOU, L.; COX, D.; KEENAM, M. P.; FLANAGAN, K.Comparativeanalysisofpublic, semi-public and recentlyprivatisedresearch centres: final project report. Manchester: Universityof Manchester, 2002.

GIANNOPOULOU, E.; BARLATIER, P. J.; PÉNIN, J. Same but different? Research and technology organizations, universities and the innovation activities of firms. Research Policy, [S. I.], v. 48, n. 1, p. 223-233, 2019.

HAMEL, G.; VALIKANGAS, L. The quest for resilience. Harvard Business Review,[S. I.], v. 81, n. 9, p. 52-65, 2003.

HARGADON, A. B. Brokering knowledge: Linking learning and innovation. Research in Organizational Behavior, [S. I.], v. 24, p. 41-85, 2002.

HARGADON, A.; SUTTON, R. I. Technology brokering and innovation in a product development firm. Administrative Science Quarterly, [S. I.], v. 42, n. 4, p. 716-749, 1997.

HIBBERT, P.; HUXHAM, C.; RING, P. S. (ed.). Managing collaborative inter-organizational relations. In: The Oxford handbook of inter-organizational relations. Oxford: Oxford University, 2008. p. 391-416.

HILLMANN, J.; GUENTHER, E. Organizational resilience: a valuable construct for management research?. International Journal of Management Reviews, [S. I.], v.23, n. 1, p. 7-44, 2021.

HOLGERSSON, M.; GRANSTRAND, O.; BOGERS, M. The evolution of intellectual property strategy in innovation ecosystems: Uncovering complementary and substitute appropriability regimes. Long Range Planning, [S. I.], v. 51, p. 303-319, 2017.

HOLLING, C. S. Resilience and stability of ecological systems. Annual Review of Ecology and Systematics, [S. I.], v. 4, n. 1, p. 1-23, 1973.

HORNE III, J.; ORR, J. Assessing behaviors that create resilient organizations. Employment Relations Today, [S. I.], v. 24, p. 29-39, 1998. 
HUIZINGH, E. K. Open innovation: State of the art and future perspectives. Technovation, [S. I.], v. 31, n. 1, p. 2-9, 2011.

LEYDESDORFF, L. The triple helix, quadruple helix,..., and an N-tuple of helices: explanatory models for analyzing the knowledge-based economy? Journal of the Knowledge Economy, [S. I.],v. 3, n. 1, p. 25-35, 2012.

LEYDESDORFF, L.; ETZKOWITZ, H. Emergence of a Triple Helix of university—industry—government relations. Science and Public Policy, [S. I.], v. 23, n. 5, p. 279-286, 1996.

LEYDESDORFF, L.; ETZKOWITZ, H. The triple helix as a model for innovation studies. Science and PublicPolicy, [S. I.], v. 25, n. 3, p. 195-203, 1998.

LINGO, E. L.; O'MAHONY, S. Nexus work: Brokerage on creative projects. Administrative Science Quarterly, [S. I.], v. 55, n. 1, p. 47-81, 2010.

LINNENLUECKE, M. K. Resilience in business and management research: A review of influential publications and a research agenda. International Journal of Management Reviews, [S. I.], v. 19, n. 1, p. 4-30, 2017.

LONG, J. C.; CUNNINGHAM, F. C.; BRAITHWAITE, J. Bridges, brokers and boundary spanners in collaborative networks: a systematic review. BMC Health Services Research, [S. I.], v. 13, n. 1, p. 158, 2013.

MARK, G.; SEMAAN, B. Resilience in collaboration: Technology as a resource for new patterns of action. In: PROCEEDINGS OF THE 2008 ACM CONFERENCE ON COMPUTER SUPPORTED COOPERATIVE WORK. San Diego: CSCW, p. 137-146, 2008.

MARTINEZ M.; Di NAUTA P.; SARNO D. Real and apparent changes of organizational processes in the era of big data analytics. [S. I.]: Franco Angeli, 2017.

MAZZOLENI, R.; NELSON, R. Public research institutions and economic catch-up. Research Policy, [S. I.], v. 36, n. 10, p. 1512-1528, 2007.

MCMANUS, S.; SEVILLE, E.; BRUNSDON, D.; VARGO, J. Resilience management: a framework for assessing and improving the resilience of organisations. New Zealand: University of Canterbury, 2007.

MODELOS institucionais das organizações de pesquisa. Brasília: Centro de Gestão e Estudos Estratégicos. 72 p. (Série Documentos Técnicos 3).

NATH, P. Knowledge management in research and technology organizations in a globalized era. Perspectives on Global Development and Technology, [S. I.], v. 7, n. 1, p. 37-54, 2008.

PETZOLD, N.; LANDINEZ, L.; BAAKEN, T. Disruptive innovation from a process view: A systematic literature review. Creativity and Innovation Management, [S. I.], v. 28, n. 2, p. 157-174, 2019.

POOLEY, J. A.; COHEN, L. Resilience: a definition in context. Australian Community Psychologist, [S. I.], v. 22, n. 1, p. 30-37, 2010.

PUFFAL, D. P.; COSTA, A. B. A interação universidade-empresa e a inovação: resenha de estudos e a situação brasileira. In: SIMPÓSIO DE GESTÃO DA INOVAÇÃO TECNOLÓGICA, 25, p. 1-15, 2008.

RADZIWON, A.; BOGERS, M.; BILBERG, A. Creating and capturing value in a regional innovation ecosystem: A study of how manufacturing SMEs develop collaborative solutions. International Journal of Technology Management, [S. I.], v. 75, n. 1-4, p. 73-96, 2017.

RITALA, P.; AGOURIDAS, V.; ASSIMAKOPOULOS, D.; GIES, O. Value creation and capture mechanisms in innovation ecosystems: a comparative case study. International Journal of Technology Management, [S. I.], v. 63, n. 3-4, p. 244-267, 2013.

RUIZ-MARTIN, C.; LOPEZ-PAREDES, A.; WAINER, G. What we know and do not know about organizational resilience. International Journal of Production Management and Engineering,[S. I.], v.6, n. 1, p. 11-28, 2018. 
SILVA, P. A política nacional de inovação e as instituições de ciência e tecnologia (ICTs). Cadernos de Prospecção, [S. I.], v. 14, n. 1, p. 1-4, 2021.

\section{Contato:}

Silvio Bitencourt da Silva

E-mail: sibitencourt@unisinos.br

Daniel Pedro Puffal

E-mail: dpuffal@unisinos.br

Taís Müller Flores

E-mail: taismflores@gmail.com 